\title{
A CASE REPORT ON TAKAYASU'S ARTERITIS
}

\author{
SHARMILA MOHAN, R. LAKSHMI*
}

Department of Pharmacy Practice, Amrita School of Pharmacy, Kochi, Amrita Vishwa Vidyapeetham, Amrita University, India Email: lakshmir@aims.amrita.edu

Received: 03 Sep 2016 Revised and Accepted: 17 Jan 2017

\begin{abstract}
Takayasu's arteritis (TA) is a rare large-vessel vasculitis that affects large arteries, mainly the aorta and its branches. It is also called a pulseless disease because of diminished or absent pulses in the upper extremities of the patient. The coronary, pulmonary and renal arteries are also affected in the progression of the disease. The prevalence of the disease is more in Asian countries and it has unknown etiopathogenesis. Here we discuss a case of TA in a $15 \mathrm{y}$ old girl who was admitted with moderate LV dysfunction. The diagnosis was carried out from the results of CT aortogram which showed stenosis of right common carotid, left subclavian, left vertebral artery, right renal artery and lower lobe pulmonary arteries and other clinical examinations. Treatment was initiated with methylprednisolone and cyclophosphamide along with symptomatic treatment. But the disease progressed with the development of complications like peripheral leg ulcers. The patient was initiated palliative care in view of altered sensorium and severe $\mathrm{LV}$ dysfunction, but the patient succumbed to a sudden cardiac arrest. Early identification and initiation of aggressive treatment can help in symptom-free survival.
\end{abstract}

Keywords: Takayasu's arteritis, Vasculitis, Coronary artery

(c) 2016 The Authors. Published by Innovare Academic Sciences Pvt Ltd. This is an open access article under the CC BY license (http://creativecommons.org/licenses/by/4. 0/) DOI: http://dx. doi.org/10.22159/ijpps.2017v9i3.15034

\section{INTRODUCTION}

Takayasu's arteritis (TA) is a rare, autoimmune, idiopathic, largevessel vasculitis that affects large arteries, mainly the aorta and its branches [1, 2]. In 1908 Takayasu, a Japanese ophthalmologist reported the first case of TA [2]. TA has been reported from different parts of the world, but the prevalence is more in oriental countries like Japan, India, Korea and Thailand [3]. The etiopathogenesis of the arthritis is not known, but studies are being conducted regarding the immunological and genetic aspects of the disease $[3,4]$. In most of the cases, irregular thickening of the walls of the aorta and its branch vessels with intimal stenosis is seen. When the aortic arch is affected, the orifices of aortic branch vessels to the upper part of the body could be narrowed markedly. The coronary, pulmonary and renal arteries are also affected in the disease process [5]. The clinical presentation of TA varies depending on the blood vessels involved. Diminished or absent pulses of upper extremities are found in most of the patients, hence also called pulse less disease [6]. Early reviews suggest that the disease is confined to the female population, but now it has been reported that males are also affected, but disease manifestations may vary among populations [2].

Here, we report a case of $15 \mathrm{y}$ old girl who was referred to our hospital for Left Ventricular (LV) dysfunction evaluation.

\section{CASE REPORT}

A 15 y old girl started to have significant weight loss and amenorrhea over few months associated with tiredness after hospitalization and treatment for high-grade fever associated with generalised arthralgia at a local hospital. For this, she was taken to a gynaecologist who attributed the symptoms to anemia and was treated with oral iron. Later the mother also noticed increased precordial activity and fast heart rates for which she was evaluated and thought to be due to anxiety. There was a history of on and off aching pain in the left upper limb. She had an episode of syncope during her exams which was again thought to be due to anxiety. Later she developed loose stools and vomiting. On fluid therapy, she developed significant chest discomfort and breathlessness and was detected to have cardiomegaly and LV dysfunction. No family history of cardiovascular diseases was documented.

She was referred to our hospital for evaluation of LV dysfunction. She was found to have moderate left ventricular dysfunction associated with some aortic irregularity on detailed echo- cardiogram. Her CT (Computed Tomography) aortogram showed stenosis of right common carotid, left subclavian, left vertebral artery and right renal artery. There was also stenosis of lower lobe pulmonary arteries. Laboratory findings showed elevated erythrocyte sedimentation rate (ESR) and C-reactive protein level at the time of admission. Arterial pulse was absent in left upper limb. Ophthalmic evaluation was suggestive of grade 1-2 hypertensive retinopathy. Considering these findings, the diagnosis was made as TA with the involvement of pulmonary arteries.

The patient was started with pulse methylprednisolone therapy $1 \mathrm{~g}$ over $30 \mathrm{~min}$ for $3 \mathrm{~d}$ followed by maintenance oral prednisolone 20 mg 1-1-1 and methotrexate injection once in a w.

After one mo, a repeat echocardiogram was done for a reassessment of LV function which showed the presence of a clot in left ventricle and patient was started on low molecular weight heparin and warfarin. Cyclophosphamide injection, once in every $3 \mathrm{w}$ was started as per rheumatology opinion. The aortic angiogram showed totally occluded left carotid, vertebral, subclavian and right carotid artery.

Five mo after diagnosis, she underwent stenting of a left renal artery in view of severe hypertension and uncontrolled LV dysfunction. Hypertension was controlled with multiple antihypertensive. She received 5 cycles of pulse cyclophosphamide injection as antiinflammatory therapy. Leg ulcers were debrided and dressed regularly. She underwent a lumbar sympathectomy for pain relief. Her ventricular function waxed and waned requiring multiple doses of Levosimendan over $6 \mathrm{w}$. The disease however progressed in severity leading to bilateral peripheral leg ulcers. Meropenem was started after the wound tissue culture showed growth of Proteus mirabilis, Klebsiellapneumoniae and Acinetobacterbaumannii organisms. The patient was shifted to intensive care unit, in view of altered sensorium and Severe LV dysfunction. The child was given palliative care, after obtaining a DNI/DNR (Do Not Intubate/Do Not Resuscitate) consent from the parents. The patient succumbed to a sudden cardiac arrest one year after diagnosis.

\section{DISCUSSION}

$\mathrm{TA}$ is a chronic, progressive, granulomatous vasculitis which commonly occurs in the second or third decade of life [2]. The most common clinical features are significantly low blood pressure (BP) and weaker pulses in the upper extremities associated with coldness or numbness of the fingers $[4,5]$. 
The clinical course of the arteritis is usually divided into an active inflammatory phase and chronic phase. The early active phase is characterised by the systemic disease with signs of fever, malaise, loss of appetite, weight loss, headaches, dizziness, arthralgia, skin rashes, etc which lasts for weeks to months. The clinical manifestations in the late, chronic phase may vary depending on the location of the arterial stenosis. The arteries involved are coronary arteries, pulmonary arteries, renal arteries, aortic arch and aortic branches and clinical manifestations include ischemic heart disease, dyspnea, ischemia of stomach and intestines, arterial hypertension, congestive heart failure, etc $[6,7]$. Our patient presented fever, headache, dizziness and arthralgia in the initial phase. In the chronic phase, the patient had an absence of radial pulses in left upper limb, hypertension, hypertensive retinopathy, LV dysfunction, peripheral leg ulcers and congestive heart failure.

According to American College of Rheumatology, at least three out of the following six criteria should be satisfied for a definite diagnosis of TA. These criteria are, age under 40 at disease onset, claudication of extremities, decreased brachial artery pulse, blood pressure difference more than $10 \mathrm{mmHg}$ between arms, a bruit over subclavian arteries or aorta and angiogram abnormalities: occlusion or narrowing in the aorta or its main branches [5].

Considering this patient, all six criteria were met. The patient also had raised ESR, CRP and anemia which reflects the underlying inflammatory process. But these laboratory investigations are usually nonspecific for diagnosis of TA. In a study conducted by Park MC et al. in South Korea, $23 \%$ of the patients showed normal laboratory results even with active disease $[5,8]$. There is no diagnostic serological test for TA and the symptoms of the disease are constitutional like fever, malaise, loss of appetite, weight loss, headaches, dizziness, arthralgia in the initial phase. This makes difficulty in early diagnosis of the disease [1].

Classification of the disease is carried out based on the involvement of blood vessels into different types as shown in table 1.

Table 1: New angiographic classification of Takayasu arteritis, Takayasu conference 1994

\begin{tabular}{ll}
\hline Type & Vessel involvement \\
\hline Type I & Branches from the aortic arch \\
Type IIa & Ascending aorta, aortic arch and its branches \\
Type IIb & Ascending aorta, aortic arch and its branches, thoracic descending aorta \\
Type III & Thoracic descending aorta, abdominal aorta, and/or renal arteries \\
Type IV & Abdominal aorta and/or renal arteries \\
Type V & Combined features of types IIb and IV \\
\hline
\end{tabular}

According to this classification system, the involvement of the coronary or pulmonary arteries should be designated as $\mathrm{C}(+)$ or $\mathrm{P}$ $(+)$, respectively $[2,9]$.

Our patient was diagnosed as TA Type $\mathrm{V}(\mathrm{P}+)$ as there was concentric wall thickening of aorta, renal artery stenosis and complete stenosis of bilateral descending pulmonary artery in CT aortogram.

Management of systemic and vascular inflammation is carried out using corticosteroids and immunosuppressive agents. Treatment may be initiated with high dose corticosteroids, such as prednisolone. Methotrexate, Cyclophosphamide, azathioprine are used for immunosuppressant. Cyclophosphamide is usually used for the treatment of systemic vasculitis when the condition is severe or lifethreatening. In chronic stages of TA, endovascular revascularization procedures or interventions like balloon angioplasty or stent placement should be considered. Procedures should be undertaken only after the suppression of inflammation in the affected arteries. Surgical procedures carry risk and success rate depends upon the location and stage of stenosis of blood vessel $[6,10]$.

Here the patient had severe hypertension and uncontrolled LV dysfunction. Hypertension usually occurs due to renal artery stenosis and poorly controlled hypertension is the cause of cardiac complications. It was managed by multiple antihypertensive and by stenting of the left renal artery. But the disease progression was fast even with the corticosteroid and immune-suppressant therapy.

\section{CONCLUSION}

Here the diagnosis was carried out only after the disease was progressed. In this case, the child had poorer outcome due to the presence of complications like hypertension, aneurysm, progressive vascular involvement and cardiac involvement. Hence, symptom-free survival is achieved only by early identification of comorbid complications like hypertension and initiation of aggressive treatment.

\section{CONFLICT OF INTERESTS}

Declared none

\section{REFERENCES}

1. Saab F, Giugliano RP, Giugliano GR. Takayasu arteritis in a young woman A 4-year case history. Tex Heart 2009;36:470-4.

2. Johnston SL, Lock RJ, Gompel MM. Takayasu arteritis: a review. J Clin Pathol 2002;55:481-6.

3. Jain S, Pondaiah SK. Takayasu's arteritis: a review of epidemiology and etiopathogenesis. Indian J Rheumatol 2015;10:S22-9.

4. Das D, Mondal KK, Ray B, Chakrabarti A. A case of unusual presentation of Takayasu's arteritis. Indian J Ophthalmol 2010;58:148-50.

5. Al-Bishri J. Takayasu's arteritis: a review article. $\mathrm{Br} \mathrm{J}$ Med Med Res 2013;3:811-20.

6. Khan MAM, Banoo H. A case report of takayasu's arteritis. Med Today 2012;24:79-81.

7. Kim HJ, Suh DC, Kim JK, Kim SJ, Lee JH, Choi CG, et al. Correlation of neurological manifestations of Takayasu arteritis with cerebral angiographic findings. Clin Imaging 2005;29:79-85.

8. Park MC, Park YB, Jung SY, Lee KH, Lee SK. Anti-endothelial cell antibodies and antiphospholipid antibodies in Takayasu's arteritis: correlations of their titers and isotype distributions with disease activity. Clin 2006;24(2, Suppl 41):S10-6.

9. Moriwaki R, Noda M, Yajima M, Sharma BK, Numano F. Clinical manifestations of Takayasu arteritis in India and Japan-new classification of angiographic findings. Angiology 1997;48:369-79.

10. Keser G, Direskeneli H, Aksu K. Management of Takayasu arteritis: a systematic review. Rheumatology (Oxford) 2014:53:793-801.

\section{How to cite this article}

- Sharmila Mohan, R Lakshmi. A case report on takayasu's arteritis. Int J Pharm Pharm Sci 2017;9(3):296-297. 